\title{
HOXA7 Promotes Metastasis in KRAS Mutant Colorectal Cancer by Regulating Myeloid-derived Suppressor Cells
}

\section{Yunzhi Dang ( $\sim$ dangyunzhi@xiyi.edu.cn )}

Shanxi Provincial Peoples Hospital https://orcid.org/0000-0002-8059-0500

Jiao Yu

Shanxi Provincial Peoples Hospital

Shuhong Zhao

Shanxi Provincial Peoples Hospital

Ximing Cao

Shanxi Provincial Peoples Hospital

Qing Wang

Shanxi Provincial Peoples Hospital

\section{Primary research}

Keywords: colorectal cancer, metastasis, HOXA7, myeloid-derived suppressor cells.

Posted Date: November 8th, 2021

DOI: https://doi.org/10.21203/rs.3.rs-1042676/v1

License: (c) (1) This work is licensed under a Creative Commons Attribution 4.0 International License.

Read Full License

Version of Record: A version of this preprint was published at Cancer Cell International on February 19th, 2022. See the published version at https://doi.org/10.1186/s12935-022-02519-9. 


\section{Abstract}

Background: KRAS mutation accounts for $30-50 \%$ of human colorectal cancer (CRC). Due to paucity of effective treatment options, KRAS mutant CRC is difficult to treat in clinic. Metastasis is still the major reason for the high mortality of KRAS mutant $\mathrm{CRC}$, but the exact mechanism remains unclear. Here, we report a novel role of Homeobox 7 (HOXA7) in promoting KRAS mutant CRC metastasis and probed therapy strategies for these subpopulation patients.

Methods: The expression of HOXA7 was detected in human CRC cohort by immunohistochemistry. The function of HOXA7 in KRAS mutant CRC metastasis was analyzed by cecum orthotopic model.

Results: The elevated expression of HOXA7 was positively correlated with lymph node metastasis, distant metastasis, poorer tumor differentiation, higher TNM stage, and poor prognosis in CRC patients. Furthermore, HOXA7 is an independent prognostic marker of KRAS mutant CRC patients $(P<0.001)$, while not for KRAS wild-type CRC patients $(P=0.575)$. HOXA7 overexpression increased the metastasis ability of KRAS mutant CT26 cell, and promoted the infiltration of MDSCs at the same time. When MDSCs infiltration was depleted by CXCR2 inhibitor, it can markedly suppress the metastasis rate in CT26 cell. The combination of CXCR2 inhibitor SB265610 and anti-programmed death-ligand 1 (anti-PD-L1) can largely inhibit metastasis in KRAS mutant CRC.

Conclusions: HOXA7 overexpression upregulated CXCL1, which promoted MDSCs infiltration. Interruption of this loop might provide a promising treatment strategy for HOXA7-mediated KRAS mutant CRC metastasis.

\section{Introduction}

Colorectal cancer (CRC) ranks as the third most commonly diagnosed cancer and the second cause of cancer mortality worldwide. The 5-year survival rate is only $12-14 \%$ in patients with metastatic CRC [1]. KRAS mutation accounts for $30-50 \%$ of human CRC, and its presence correlates positively with disease aggressiveness and metastasis [2, 3]. Anti-EGFR antibodies, such as cetuximab or panitumumab, in combination with chemotherapy are effective therapeutic approaches for metastatic CRC patients with KRAS wild-type. However, the clinical efficacy for KRAS mutant CRC patients is often poor due to the intrinsic drug resistance by KRAS mutation [4]. In recent years, immunotherapy exhibited excellent efficacy for CRC, while it shows poor response for KRAS mutant CRC, emphasizing treatment of these subpopulation patients remains a challenge. Recently, the combination of AMG510 and anti-PD-1 contributes durable anti-tumor response[5], which indicates that combination therapy may provide a promising treatment option for KRAS mutant CRC subpopulations.

The Homeobox (HOX) family of transcription factors is known to play key roles in embryonic development and regulates many aspects of cellular processes [6]. In recent years, mounting evidence has showed that dysregulation of HOX gene facilities cancer initiation and progression through different mechanism [7]. In mammals, HOX family comprises 39 proteins, which was classified to four separate 
clusters (A, B, C and D). HOXA subfamily genes are critical regulators in cancer metastasis. Several studies reported that HOXA7, a member of HOXA subfamily, function as an oncogene and contributes to malignant behaviors in kinds of human cancer, including hepatocellular carcinoma [8], laryngeal squamous cell cancer [9], ovarian cancer [10]. However, little is known about the role of HOXA7 in CRC metastasis.

Immunosuppression observed during cancer development and progression is a result of the orchestration of many cell types, including MDSCs. The accumulation of relatively immature and pathologically activated MDSCs with potent immunosuppressive activity is common in tumors. Mounting data have shown the accumulation of MDSCs, which induce local and possibly immunosuppression, is correlated with tumor progression [11]. In addition, MDSCs can directly promote tumor cell survival, angiogenesis, invasion and metastasis [12]. MSI-L CRC is more extensively infiltrated by Tregs and MDSCs, while T cells are significantly reduced, which is considered to be the main reason for the poor efficacy of immune checkpoint blocking $[13,14]$. Previous study had revealed that for KRAS mutant CRC and KRAS wild types $\mathrm{CRC}$, there is a significant difference in infiltration of immune cells and expression of immune-related molecules. For example, KRAS mutant CRC displays the feature of reduced T helper 1 (Th1)-centric coordinated immune response cluster, and reduced infiltration of cytotoxic cells [15]. The MDSCs infiltration was significantly increase, and depleted MDSCs can significantly improve the efficacy of antiPD-L1 116,17$]$. Whereas the haptic ongenic signal in KRAS mutant CRC that drives MDSCs recruitment and activation remains poorly understood.

Here, we demostrated that HOXA7 was upregulation and associated with poor prognosis in KRAS mutant CRC. Ovexpression of HOXA7 promoted KRAS mutant CRC metastasis by upregulating CXCL1. The administration of CXCR2 inhibitor SB265610 and anti-PD-L1 markedly suppressed HOXA7-mediated KRAS mutant CRC metastasis.

\section{Materials And Methods}

\section{Immunohistochemistry}

This study was approved by the ethics the Committee of Shaanxi Provincial People's Hospital, and informed consent was written and based on the ethical guidelines of the 1975 Declaration of Helsinki. In addition, the privacy rights of human subjects were always observed. CRC specimens and matched adjacent tissues were used to construct a tissue microarray (Shanghai Biochip Co, Ltd. Shanghai, China). The tissue microarray was stained for HOXA7 (Abcam, ab211521), CD11b (Abcam, ab6640)

CD8(Abcam, ab4055) expression. Immunohistochemistry was performed on 4- $\mu m$ thick, routinely processed paraffin-embedded sections. Images were obtained under a light microscope (Olympus, Japan) equipped with a DP70 digital camera.

Analyses were performed by two independent observers who were blinded to the clinical outcome. The immunostaining intensity was scored on a scale of 0 to 3:0 (negative), 1 (weak), 2 (medium) or 3 
(strong). The percentage of positive cells was evaluated on a scale of 0 to 4 : 0 (negative), 1 (1\%-25\%), 2 (26\%-50\%), 3 (51\%-75\%), or 4 (76\%-100\%). The final immuno-activity scores were calculated by multiplying the above two scores, resulting an overall score which range from $0 \sim 12$. Each case was ultimately considered "negative" if the final score ranges from $0 \sim 3$, and "positive" if the final score ranges from 4 12.

\section{Animal experiment}

All the mice were male. Luciferase labeled mouse CRC cells $\left(4.0 \times 10^{6}\right)$ were injected into the cecal wall in nude or BABL/C mice under anesthesia (10 for each group). We monitor the tumor formation and metastasis weekly using the IVIS-100 Imaging System. At the 9 weeks, the mice were sacrificed and the livers and lungs were collected and underwent histological examination.

\section{Statistical analysis}

Statistics were calculated with SPSS software (version 20.0). P values were statistically analyzed by the $\chi 2$ test for categorical variables and by Student's test for quantitative data. The recurrence and survival data were analyzed by the Kaplan-Meier method. Cox proportional hazards model was used for univariate and multivariate analyses. Differences were considered statistically significant when $p<0.05$.

\section{Results}

\section{Elevated HOXA7 is associated with poor prognosis in CRC patients harboring KRAS mutation}

To characterize the function of HOXA7 in CRC, we examined its mRNA expression in 20 normal colorectal epithelial specimens, and 100 paired CRC and adjacent nontumor specimens. We found that the CRC tissues displayed higher HOXA7 mRNA level than paired nontumor tissues and normal colorectal epithelial specimens (Figure 1A, left). To determine the relationship of between HOXA7 expression with KRAS mutation status, we performed KRAS mutation test and found $46 \%$ (46 of 100) of cases harboring mutant KRAS. Notably, the HOXA7 mRNA level was significantly higher in patients with KRAS mutation than in patients with KRAS wild-type (Figure 1A, right). We then investigated the expression level of HOXA7 in established human CRC cells, and found that HOXA7 expression was higher in KRAS mutant CRC cell lines (SW620, HCT116, and Lovo) than in KRAS wild-type CRC cell lines (HT29, CaCo-2 and HCA7) (Figure 1B).

In order to evaluate the clinal significance of HOXA7 in CRC patient survival and its relationship with KRAS mutational status, we set out to profile the expression of HOXA7 using a tissue microarray consisting of $362 \mathrm{CRC}$ samples. The immunohistochemical images showed dramatically higher HOXA7 expression in KRAS mutant CRC tissues than in KRAS-wild CRC tissues (Figure 1C-D). The elevated HOXA7 expression was positively correlated with tumor differentiation, lymph node metastasis, distant metastasis, higher tumor nodule metastasis (TNM) stage and KRAS mutation (Table 1). Multivariate 
analysis indicated that overexpression of HOXA7 was an independent predictor for shorter overall survival (Table 2). Stratification the cohort into KRAS mutant cases and KRAS wild-type cases revealed that HOXA7 is an independent prognostic marker in KRAS mutant CRC $(P<0.001$, Supplementary Tale 1), while not in KRAS wild-type CRC ( $P=0.575$, Supplementary Tale 2). Furthermore, Kaplan-Meier curve showed that patients with positive HOXA7 expression had reduced overall survival time compared to these with negative HOXA7 expression. In addition, stratification analysis showed that HOXA7 expression was associated with poor survival in KRAS mutant CRC patients, but not in those with wild-type KRAS CRC patients (Figure 1E). All these works indicates that HOXA7 overexpression was a specifically prognostic predictor in KRAS mutant CRC. 
Table 1

Correlation between HOXA7 expression and clinicopathological characteristics of CRC in cohorts of human CRC issues

\begin{tabular}{|c|c|c|c|c|}
\hline \multicolumn{2}{|c|}{ Clinicopathological variables } & \multicolumn{2}{|c|}{ Tumor HOXA7 expression } & \multirow[t]{2}{*}{$P$ Value } \\
\hline & & Negative $(n=203)$ & Positive $(n=159)$ & \\
\hline \multirow[t]{2}{*}{ Age } & $\leq 50$ & 26 & 28 & 0.203 \\
\hline & $>50$ & 177 & 131 & \\
\hline \multirow[t]{2}{*}{ Gender } & female & 88 & 73 & 0.626 \\
\hline & male & 115 & 86 & \\
\hline \multirow[t]{2}{*}{ Tumor size } & $\leq 5 \mathrm{~cm}$ & 90 & 64 & 0.435 \\
\hline & $>5 \mathrm{~cm}$ & 113 & 95 & \\
\hline \multirow[t]{2}{*}{ Tumor differentiation } & well or moderate & 158 & 91 & $<0.001$ \\
\hline & poor & 45 & 68 & \\
\hline \multirow[t]{2}{*}{ Tumor invasion } & T1-T3 & 161 & 116 & 0.157 \\
\hline & $\mathrm{T} 4$ & 69 & 103 & \\
\hline \multirow[t]{2}{*}{ Lymph node metastasis } & absent & 177 & 117 & 0.001 \\
\hline & present & 22 & 17 & \\
\hline \multirow[t]{2}{*}{ Distant metastasis } & absent & 134 & 56 & 0.001 \\
\hline & present & 69 & 103 & \\
\hline \multirow[t]{2}{*}{ TNM stage } & $\mathrm{I}-\mathrm{II}$ & 131 & 59 & $<0.001$ \\
\hline & III-IV & 73 & 100 & \\
\hline \multirow[t]{2}{*}{ KRAS status } & Wild & 140 & 68 & $<0.001$ \\
\hline & Mutation & 63 & 91 & \\
\hline
\end{tabular}


Table 2

Univariate and multivariate analysis of factors associated with survival and recurrence in cohort of human CRC (Overall Population)

\begin{tabular}{|c|c|c|c|c|}
\hline \multirow[t]{2}{*}{ Clinical Variables } & \multicolumn{2}{|c|}{$\begin{array}{l}\text { Univariate COX regression } \\
\text { analysis }\end{array}$} & \multicolumn{2}{|c|}{$\begin{array}{l}\text { Multivariate COX regression } \\
\text { analysis }\end{array}$} \\
\hline & $\mathrm{HR}(95 \% \mathrm{Cl})$ & $\begin{array}{l}P \\
\text { value }\end{array}$ & $\mathrm{HR}(95 \% \mathrm{Cl})$ & $\begin{array}{l}P \\
\text { value }\end{array}$ \\
\hline Age $(\leq 50$ versus $>50)$ & $\begin{array}{l}1.065(0.750- \\
1.512)\end{array}$ & 0.726 & & \\
\hline Gender (female vs male) & $1.142(0.885-686)$ & 0.349 & & \\
\hline Tumor size $(\leq 5 \mathrm{~cm}$ vs $>5 \mathrm{~cm})$ & $\begin{array}{l}1.278(0.985- \\
1.658)\end{array}$ & 0.065 & & \\
\hline $\begin{array}{l}\text { Tumor differentiation (well/moderate } \\
\text { vs poor) }\end{array}$ & $\begin{array}{l}0.185(0.141- \\
0.242)\end{array}$ & $<0.001$ & $\begin{array}{l}0.733(0.522- \\
1.030)\end{array}$ & 0.073 \\
\hline Tumor invasion (I-II versus III) & $\begin{array}{l}0.234(0.262- \\
0.455)\end{array}$ & $<0.001$ & $\begin{array}{l}0.570(0.420- \\
0.774)\end{array}$ & $<0.001$ \\
\hline $\begin{array}{l}\text { Lymph node metastasis (absent vs } \\
\text { present) }\end{array}$ & $\begin{array}{l}0.122(0.070- \\
0.132)\end{array}$ & $<0.001$ & $\begin{array}{l}0.560(0.313- \\
1.002)\end{array}$ & 0.051 \\
\hline $\begin{array}{l}\text { Distant metastasis (absent vs } \\
\text { present) }\end{array}$ & $\begin{array}{l}0.155(0.114- \\
0.211)\end{array}$ & $<0.001$ & $\begin{array}{l}0.508(0.351- \\
0.737)\end{array}$ & $<0.001$ \\
\hline TNM stage (I/II vs III/IV) & $\begin{array}{l}0.096(0.114- \\
0.211)\end{array}$ & $<0.001$ & $\begin{array}{l}0.232(0.131- \\
0.412)\end{array}$ & $<0.001$ \\
\hline $\begin{array}{l}\text { HOXA7 expression (negative vs } \\
\text { positive) }\end{array}$ & $\begin{array}{l}0.418(0.322- \\
0.541)\end{array}$ & $<0.001$ & $\begin{array}{l}0.724(0.552- \\
0.948)\end{array}$ & 0.019 \\
\hline
\end{tabular}

\section{Elevated expression of HOXA7 promotes KRAS mutant CRC metastasis in immunocompetent mice}

To understand the function of HOXA7 in KRAS mutant CRC cells, we evaluated the effect of HOXA7 on cell invasion and migration in a loss of function experiments in vitro and in vivo. Knockdown of HOXA7 failed to suppress the invasion and migration ability of SW620 cells (Supplementary Figure1A). Furthermore, knockdown of HOXA7 did not alter the metastasis rate of SW620 cells in orthotopic transplantation tumor in nude mice (Supplementary Figure 1B-D). These results indicated that HOXA7 expression is not crucial for migration abilities of CRC in the immune-deficiency nude mice.

In fact, CRC most commonly develop due to underlying chronic colon inflammation and altered immune response. Thus, we postulated that HOXA7 promoted CRC progression by changing the immune microenvironment. We stably upregulated the HOXA7 expression by lentivirus in CT26 cell line, which bears mutant KRAS. Western blot analyses showed that endogenous HOXA7 was overexpression in CT26 cells (Figure 2A). To further study the function of HOXA7 in KRAS mutant CRC metastasis, we performed the cecum orthotopic tumor implantation experiment in BABL/C mice. It showed that HOXA7 
overexpression can increase the intensity of bioluminescent imaging signal (Figure 2B-C). Histological analysis confirmed that upregulated of HOXA7 can increase the liver and lung metastasis rate, as well as its metastatic nodules' number (Figure 2E-G). In addition, HOXA7 can shorten the overall survival of $B A B L / C$ mice (Figure 2D). These results suggested that overexpression of HOXA7 is essential for KRAS mutant CRC metastasis in immunocompetent mice.

\section{HOXA7 promotes KRAS mutant CRC metastasis by recruitment of MDSCs}

To decipher the underling mechanisms by which HOXA7 signaling promotes KRAS mutant CRC metastasis, we investigated the cellular immune response. The percentage of tumor infiltrating immune and inflammatory cells were assessed by flow cytometric analysis. The transplantation of CT26-HOXA7 cells significantly increased MDSCs (marked by CD45+/CD11b/Gr1+) infiltration, and decreased accumulation of CD8+T cells (marked by CD45+/CD3+/CD8+) compared with CT26-control cell. In contrast, the percentage of other immune cells, such as CD4+T cell, regulatory $\mathrm{T}(\mathrm{Treg})$ cells and TAMs immune cells did not change significantly (Figure 3A-B). In addition, IHC staining showed that MDSCs infiltration markedly elevated, while CD8+ T cells infiltration decreased in CT26-HOXA7 tumors (Figure 3C). We further evaluated the expression of HOXA7, CD11b and CD8 in KRAS mutant CRC cohort. Consistent with the above results, IHC staining revealed that HOXA7 expression positively associated with CD11b upregulation, while negatively associated with CD8 expression in KRAS mutant CRC patients (Figure 3E).

\section{HOXA7 overexpression induces MDSCs chemotaxis through HOXA7-CXCL1 in KRAS mutant CRC}

To study the underlying mechanism of CRC recruit MDSCs, we selected the KRAS mutant SW620 CRC cell lines with high expression of HOXA7 and constructed SW620-shHOXA7 stable cell lines by lentivirus. We compared transcriptome changes in SW620-shHOXA7 and SW620-shcontrol cells using a Affymetrix PrimeView Human Gene Expression Array. Downregulation of HOXA7 reduced the expression of several metastasis-related genes, such as CXCL1, OPN and CXCR2 (Supplementary Table S3). Considering the important role of CXCL1 in cancer progression, we focused on CXCL1 for further study. CXCL1 interacts with its receptor CXCR2 to recruit MDSCs $[18,19]$. Previous studies indicated that tumor cell-derived CXCL1 promotes the recruitment and infiltration of MDSCs to the tumor site and facilitates CRC metastasis [11]. We hypothesized whether HOXA7 promoting MDSCs infiltration by upregulating CXCL1.

Real time assay showed that downregulation of HOXA7 markedly reduced CXCL1 expression in SW620 and HCT116 cell (Figure 4A). It has been reported CXCL1 can regulate the accumulation of MDSCs. To study the function of HOXA7 in CRC metastasis, we downregulated CXCL1 in CT26 cell by lentivirus and generated the cecum orthotopic tumor implantation model in immune competent BABL/C mice. In vivo study showed that CXCL1 knockdown can significantly reduce lung and liver metastasis, while prolonged 
the survival of CT26-HOXA7 group compared to the control group (Figure 4B-E). Moreover, IHC staining and Flowcytometric analysis exhibited that knockdown of CXCL1 expression in CT26-HOXA7 cell can significantly reduce the infiltration rate of MDSCs, while increase the CD8+ T cells infiltration compared with control (Figure 4E-F). Taken together, these results showed that HOXA7 promote KRAS mutant CRC metastasis and MDSCs chemotaxis through HOXA7-CXCL1 axis.

\section{Depletion of MDSCs decreases HOXA7-mediated KRAS mutant CRC metastasis}

Enrichment of MDSCs in CT26-HOXA7 cells orthotopic transplantation tumors prompted us to study the specific role of MDSCs in HOXA7-mediated KRAS mutant CRC metastasis. We depleted the MDSCs by neutralizing monoclonal antibody (clone RB6-8C5), a well characterized anti-Gr1 [20]. In vivo metastasis assay showed that HOXA7 upregulation promoted lung and liver metastasis and reduced survival time of $\mathrm{BABL} / \mathrm{C}$ mice. Anti-Gr1 decreased the incidence of lung and liver metastasis burden and prolonged overall survival time in CT26-HOXA7 cells group (Figure 5A-E). Together, these results strongly support the view that MDSCs played a pivotal role in HOXA7-mediated KRAS mutant CRC metastasis.

\section{Combined application of CXCR2 inhibitor SB265610 and anti-PD-L1 dramatically blocks HOXA7-mediated KRAS mutant CRC metastasis}

It was reported that depletion of MDSCs showed synergistic effect with anti-PD-L1 in CRC [21, 22]. Previous studies reported that SB265610, a specific CXCR2 inhibitor, can inhibit the recruitment and infiltration of MDSCs and TAMs in CRC [16], We wondered whether SB265610 can enhance CRC response to anti-PD-L1 blockade in KRAS mutant CRC. In vivo metastatic assay demonstrated that SB265610 or anti-PD-L1 treatment alone can partially impaired the lung and liver metastasis rate and metastatic nodules, with prolonged the overall survival time of mice in CT26-HOXA7 group. Whereas combination of SB265610 and anti-PD-L1 significantly decreased the lung and liver metastasis rate and metastatic nodules, with largely prolonged survival time as compared to SB265610 or anti-PD-L1 alone (Figure 6AE).

To investigate the underlying mechanism of the antitumor response triggered by the combination SB265610 and anti-PD-L1 antibody, we examined the infiltration of MDSCs and CD8+T cells in CT26HOXA7 orthotopic CRC tumors. IHC staining showed a significantly decreased MDSCs infiltration and increased CD8+ $T$ cell infiltration $T$ cell in the combination treatment group (Figure 6F). These results indicated that double blockade of PD-L1 and SB265610 improved antitumor response and inhibited HOXA7-mediated KRAS mutant CRC metastasis.

\section{Discussion}

Despite compelling evidences supporting a role of mutant KRAS in CRC progression, intensive efforts have failed to identify effective molecular targets for this subset of CRC. A better understanding of the 
oncogenic genes may help to acquire knowledge of molecular mechanism in KRAS mutant CRC metastasis and exploit more potent combination-based therapies [16]. In the present study, we found that HOXA7 was overexpression in CRC and high levels of HOXA7 were positively associated with a higher metastasis rate, a more aggressive tumor phenotype and shorter overall survival. In addition, HOXA7 overexpression was independent poor prognostic factor in KRAS mutant CRC, but not in those with wildtype KRAS. Furthermore, HOXA7 promoted KRAS mutant CRC metastasis in immune-competent mice. These results demonstrated the importance of HOXA7 in promoting KRAS mutant CRC metastasis.

Immune invasion is one of the major hallmarks of cancers [23], often accomplished by expansion of PD1/PD-L1 axis or the infiltration of immunosuppressive cells such as MDSCs[24, 25]. MDSCs represent the universal immunosuppressive population in many pathologic conditions including cancer [26].

Accumulating evidence in recent years has even highlighted the expansion of MDSCs in human CRC as a major barrier to antitumor immunity and escape immune surveillance [27]. More importantly, MDSCs can in turn suppress CD8+ T cell cytotoxicity. In this study, we demonstrated that HOXA7 overexpression leads to immune suppression in KRAS mutant CRC via the chemotaxis of MDSCs. In vivo study showed that pharmacological depletion of MDSCs with anti-Gr1 can inhibit HOXA7-medicated KRAS mutant CRC metastasis. These findings support the view that depletion of MDSCs treatment can suppress KRASmutant CRC metastasis.

AMG510, an inhibitor of KRAS ${ }^{G 12 C}$, showed lasting antitumor response in immunocompetent mice, but its antitumor effect is weak in immunodeficient mice, indicating that T cells are necessary for AMG510 to play its role. The combination of AMG510 and anti-PD-1 can increase the infiltration of CD8+ T cells and produce durable anti-tumor response [5]. These studies have laid the foundation for the combination of KRAS inhibitors and immunotherapy, but KRASG ${ }^{12 C}$ is a rare type in CRC, accounting for only about $3 \%$ of all mutations. Most patients with KRAS mutations in CRC cannot benefit from this combination therapy. Therefore, it has become urgent need to investigate the combinate strategies to enhance treatment efficacy for KRAS-mutant CRC. In particular, the combination of anti-PD-1/anti-PD-L1 with anti-CTLA4 agents, locoregional therapies or VEGF/VEGFR inhibitors synergistically enhances antitumor immunity [28-30], indicating that antitumor agents which can activate tumor immunity, combinate with anti-PD1/anti-PD-L1 may show promising results. Our in vivo data indicated that combined treatment of SB265610 with anti-PD-L1 can dramatically inhibited HOXA7-mediated KRAS mutant CRC metastasis compared with control or single agent alone. In addition, the combination treatment significantly inhibited MDSCs cells recruitment and increased the infiltration of CD8+ T cells. These results provided a new combinational treatment strategy to inhibit HOXA7-induced KRAS mutant CRC metastasis.

In summary, we demonstrated that HOXA7 overexpression upregulated CXCL1 expression, which facilitated the recruitment and infiltration of MDSCs to KRAS mutant CRC tumor niche. Combined targeting CXCL1 and an-PD-L1 largely suppressed HOXA7-mediated KRAS mutant CRC metastasis. Our works indicated that HOXA7 was a potential prognostic biomarker for KRAS mutant CRC, and targeting the oncogenic loop may provide a promising therapy strategy for HOXA7-mediated KRAS mutant CRC metastasis. 


\section{Abbreviations}

CRC

Colorectal cancer

HOXA7

homeobox A7

HE

hematoxylin and eosin

$\mathrm{IHC}$

immunohistochemistry

BLI

bioluminescent imaging

CXCL12

chemokine (C-X-C motif) ligand 12.

\section{Declarations}

\section{Ethics approval and consent to participate}

All animal studies were approved by the Committee on the Use of Live Animals in Teaching and Research, Shaanxi Provincial People's Hospital. The study protocol was performed in accordance with the guidelines outlined in the Declaration of Helsinki. The Ethics Committee of Shaanxi Provincial People's Hospital approved the study.

\section{Consent for publication}

All the authors have actively participated in the study and have read and approved the submitted manuscript.

\section{Availability of data and materials}

The datasets used during the current study are available from the corresponding author on reasonable request.

\section{Competing interests}

The authors have declared that no competing interest exists.

\section{Funding}

This study was supported by Scientific and Technological Talents support Program Foundation of Shaanxi Provincial People's Hospital (No.2021JY-05).

\section{Authors' contributions}


Yunzhi Dang performed the experiments. Jiao Yu assisted in immunohistochemical staining and animal experiments. Shuhong Zhao provided assistance in collecting tissue samples. Ximing Cao and Qing Wang provided assistance in conceiving experiments and analyzing data. Yunzhi Dang designed the studies and wrote the paper.

\section{Acknowledgements}

We appreciated all people involved this study.

\section{References}

1. Siegel RL, Miller KD, Fedewa SA, Ahnen DJ, Jemal A: Colorectal cancer statistics, 2017. Ca A Cancer Journal for Clinicians 2017, 67(3):104-117.

2. Parker BS, Rautela J, Hertzog PJ: Antitumour actions of interferons: implications for cancer therapy. Nature Reviews Cancer 2016, 16(3):131.

3. Pereira A, Rego J, Morris V, Overman MJ, Kopetz S: Association between KRAS mutation and lung metastasis in advanced colorectal cancer. Br J Cancer 2015, 112(3).

4. Benvenuti S, Sartore-Bianchi A, Nicolantonio FD, Zanon C, Moroni M, Veronese S, Siena S, Bardelli A: Oncogenic Activation of the RAS/RAF Signaling Pathway Impairs the Response of Metastatic Colorectal Cancers to Anti-Epidermal Growth Factor Receptor Antibody Therapies. Cancer Research 2007, 67(6):2643-2648.

5. Canon J, Rex K, Saiki AY, Mohr C, Lipford JR: The clinical KRAS(G12C) inhibitor AMG 510 drives antitumour immunity. Nature 2019, 575(7781).

6. Ruddle FH, Bartels JL, Bentley KL, Kappen C, Pendleton JW: Evolution of Hox Genes. Annual Review of Genetics 1994, 28(1):423-442.

7. Shah N, Sukumar S: The Hox genes and their roles in oncogenesis. Nature Reviews Cancer 2010, 10(5):361-371.

8. Tang B, Qi G, Sun X, Tang F, Yuan S, Wang Z, Liang X, Li B, Yu S, Liu J: HOXA7 plays a critical role in metastasis of liver cancer associated with activation of Snail. Molecular Cancer 2016, 15(1):1-12.

9. Xu Y, Wang J, Zhou C: Expression Profile and Prognostic Values of HOXA Family Members in Laryngeal Squamous Cell Cancer. Social Science Electronic Publishing.

10. Wang S, Diao YJ, Zhu BB: MiR-193a-5p suppresses cell proliferation and induces cell apoptosis by regulating HOXA7 in human ovarian cancer. Neoplasma 2020, 67(4).

11. Yi-Ming, Li, Zhen-Yu, Liu, Jian-Chao, Wang, Jing-Min, Yu, Zhu-Chun, Hai-Jiao: RIP3 deficiency recruits myeloid-derived suppressor cells to hepatocellular carcinoma through the CXCL1-CXCR2 axis. Hepatology (Baltimore, Md) 2019.

12. Gabrilovich DI, Ostrand-Rosenberg S, Bronte V: Coordinated regulation of myeloid cells by tumours. Nature Reviews Immunology. 
13. Overman MJ, Mcdermott R, Leach JL, Lonardi S, André T: Nivolumab in patients with metastatic DNA mismatch repair-deficient or microsatellite instability-high colorectal cancer (CheckMate 142): an open-label, multicentre, phase 2 study. Lancet Oncology 2017, 18(9).

14. Anaricin T: PD-1 blockade in tumors with mismatch-repair deficiency. Colon \& Rectum 2015.

15. Lal N, White BS, Goussous G, Pickles OJ, Mason M, Beggs AD, Taniere P, Willcox BE, Guinney J, Middleton G: KRAS mutation and Consensus Molecular Subtypes 2 and 3 are independently associated with reduced immune infiltration and reactivity in colorectal cancer. Clinical Cancer Research An Official Journal of the American Association for Cancer Research 2017:clincanres.1090.2017.

16. Liao W, Overman MJ, Boutin AT, Shang X, Zhao D, Dey P, Li J, Wang G, Lan Z, Li J: KRAS-IRF2 Axis Drives Immune Suppression and Immune Therapy Resistance in Colorectal Cancer. Cancer Cell 2019.

17. Ganesh K, Stadler ZK, Cercek A, Mendelsohn RB, Shia J, Segal NH, Diaz LA: Immunotherapy in colorectal cancer: rationale, challenges and potential. Nature Reviews Gastroenterology \&\#38 Hepatology 2019.

18. Wang D, Sun H, Wei J, Cen B, Dubois RN: CXCL1 Is Critical for Premetastatic Niche Formation and Metastasis in Colorectal Cancer. Cancer research 2017:3655.

19. Taki M, Abiko K, Baba T, Hamanishi J, Yamaguchi K, Murakami R, Yamanoi K, Horikawa N, Hosoe Y, Nakamura E: Snail promotes ovarian cancer progression by recruiting myeloid-derived suppressor cells via CXCR2 ligand upregulation. Nature Communications 2018, 9(1):1685.

20. Pekarek, A. L: Inhibition of tumor growth by elimination of granulocytes. Journal of Experimental Medicine, 181(1):435-440.

21. Liu M, Zhou J, Liu X, Feng Y, Yang W, Wu F, Cheung OK-W, Sun H, Zeng X, Tang W: Targeting monocyte-intrinsic enhancer reprogramming improves immunotherapy efficacy in hepatocellular carcinoma. Gut 2019.

22. Zhou J, Liu M, Sun H, Feng Y, Xu L, Chan AWH, Tong JH, Wong J, Chong CCN, Lai PBS: Hepatomaintrinsic CCRK inhibition diminishes myeloid-derived suppressor cell immunosuppression and enhances immune-checkpoint blockade efficacy. Gut:gutjnl-2017-314032.

23. D H, RA W: Hallmarks of cancer: the next generation. Cell 2011, 144(5):646-674.

24. Rebekka W, Viktor F, Hu X, Vasyl N, Christopher G, Peter A, Jochen U, Viktor U: Myeloid-Derived Suppressor Cells Hinder the Anti-Cancer Activity of Immune Checkpoint Inhibitors. Frontiers in Immunology 2018, 9:1310.

25. K A, M M, J H, Y Y, N M, T B, K Y, R M, A Y, B K et al: PD-L1 on tumor cells is induced in ascites and promotes peritoneal dissemination of ovarian cancer through CTL dysfunction. Clinical cancer research : an official journal of the American Association for Cancer Research 2013, 19(6):13631374.

26. Veglia F, Perego M, Gabrilovich D: Myeloid-derived suppressor cells coming of age. Nature Immunology 2018, 19(2):108. 
27. A New Population of Myeloid-Derived Suppressor Cells in Hepatocellular Carcinoma Patients Induces CD4+CD25+Foxp3+ T Cells. Gastroenterology, 135(1):234-243.

28. Wolchok JD, Chiarion-Sileni V, Gonzalez R, Rutkowski P, Grob JJ, Cowey CL, Lao CD, Wagstaff J, Schadendorf D, Fe Rrucci PF: Overall Survival with Combined Nivolumab and Ipilimumab in Advanced Melanoma. New England Journal of Medicine 2017, 377(14):1345.

29. Victor TS, Rech AJ, Maity A, Rengan R, Minn AJ: Radiation and Dual Checkpoint Blockade Activates Non-Redundant Immune Mechanisms in Cancer. Nature 2015, 520(7547).

30. Anti-PD-1 Antibody SHR-1210 combined with Apatinib for Advanced Hepatocellular Carcinoma, Gastric or Esophagogastric Junction Cancer: An Open-label, Dose Escalation and Expansion Study. Clinical cancer research : an official journal of the American Association for Cancer Research 2018.

\section{Figures}


A

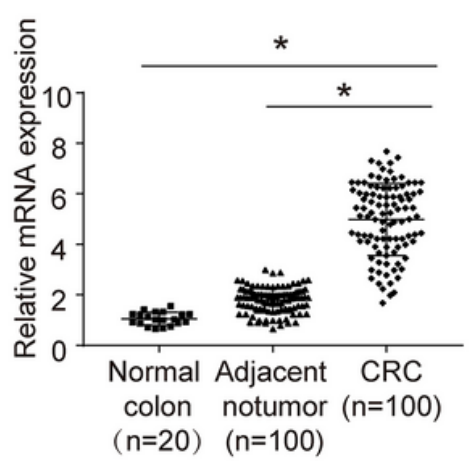

C

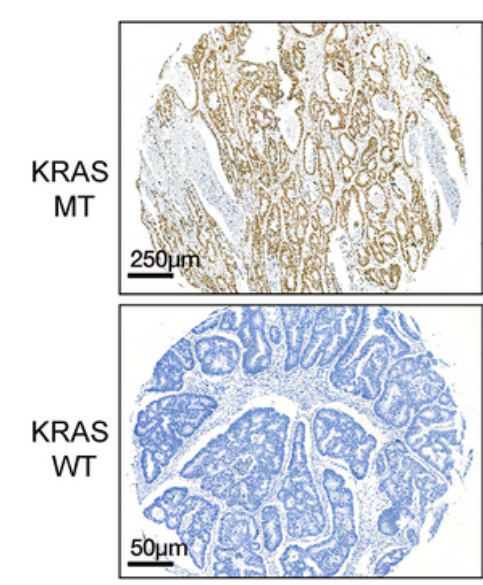

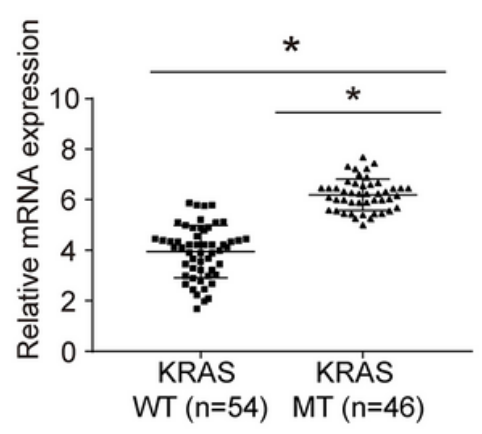

B

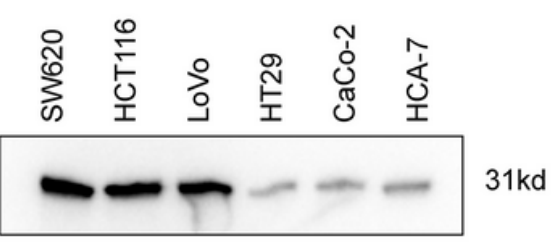

$\begin{array}{llllll}1.0 & 1.0 & 1.0 & 0.2 & 0.2 & 0.3\end{array}$

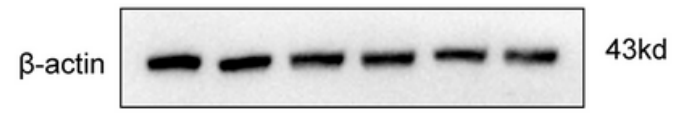

E

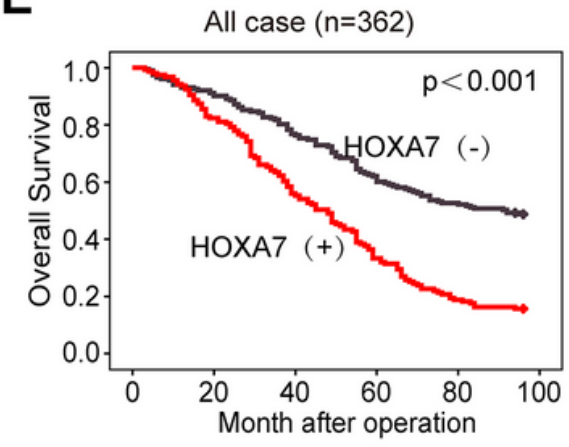

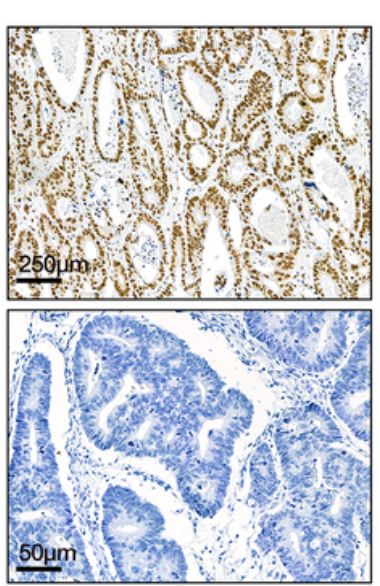
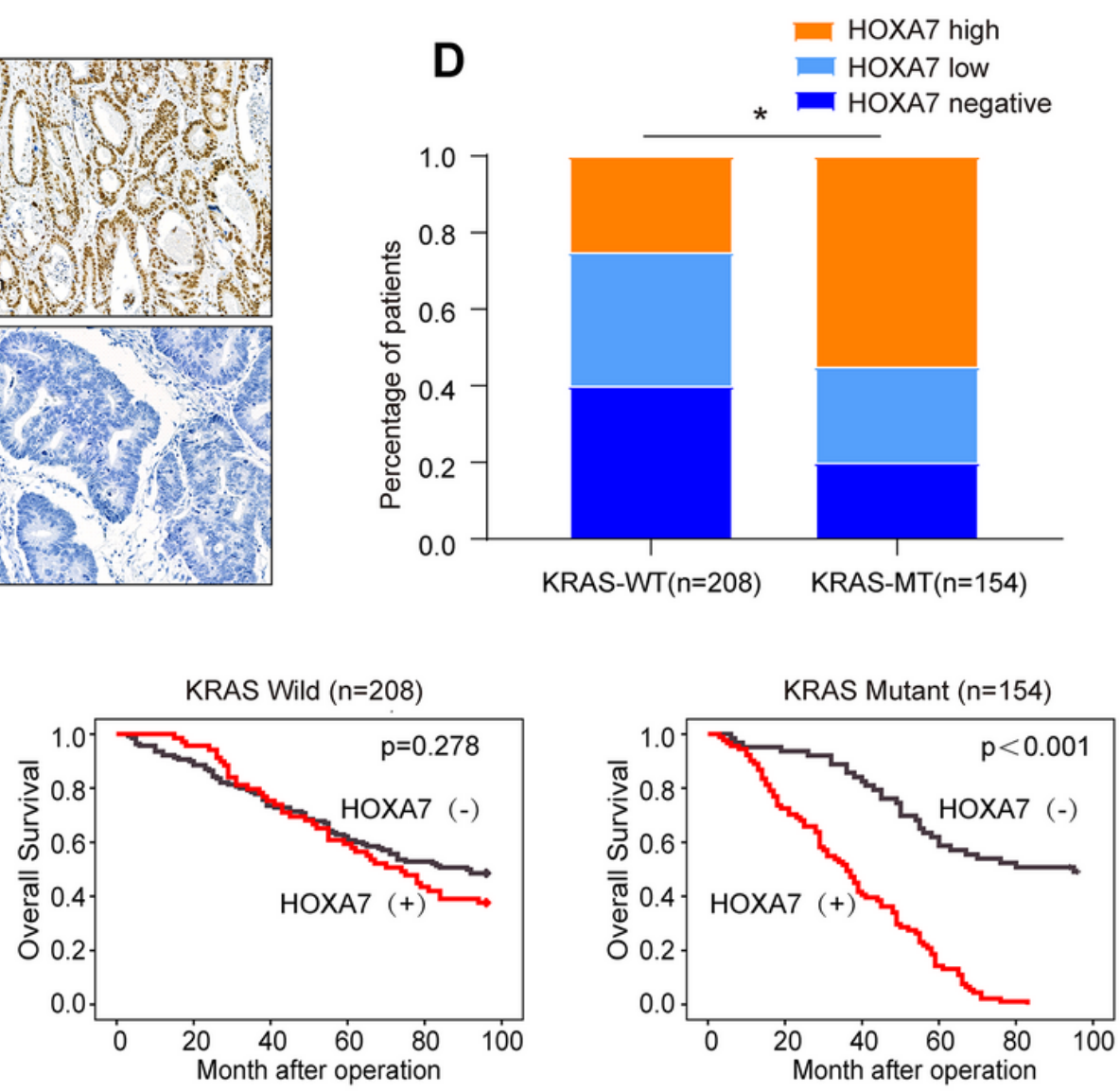

Figure 1

Elevated HOXA7 is associated with poor prognosis in KRAS mutant CRC. (A). Relative HOXA7 mRNA expression in 20 normal colon tissues and 100 paired CRC and adjacent nontumorous tissues (left). Relative HOXA7 mRNA expression in CRC tissues with KRAS wild-type or KRAS mutation (right); (B). Western blotting analysis of HOXA7 expression in KRAS mutant CRC cell lines (SW620, HCT116 and LOVO) and KRAS wild-type CRC cell lines (HT29, Caco-2, HCA-7). (C-D). Representative images of IHC staining and correlation analysis of HOXA7 expression and KRAS mutant status in CRC. (E). Kaplan-Meier analysis of the correlation of HOXA7 expression with overall survival in human CRC cohort with KRAS mutant or KRAS wild-type. 

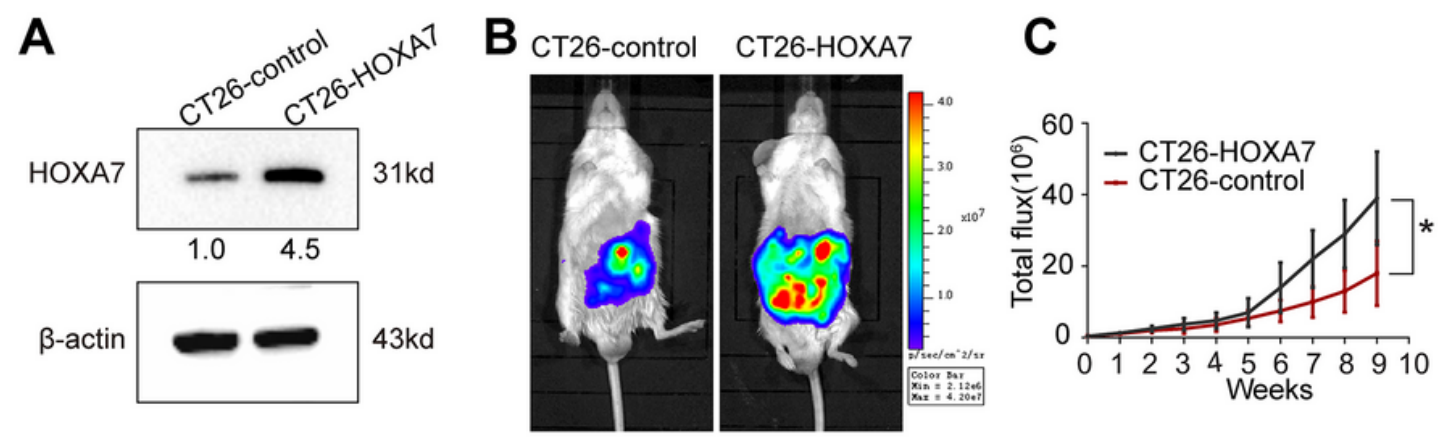

D
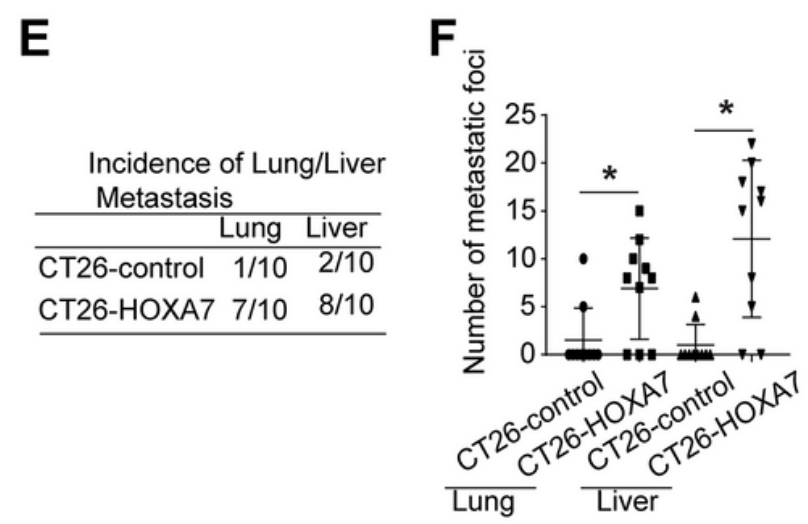

G
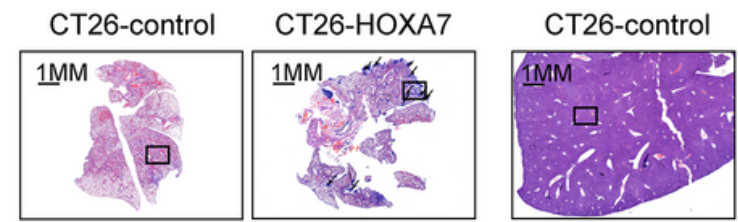

CT26-HOXA7
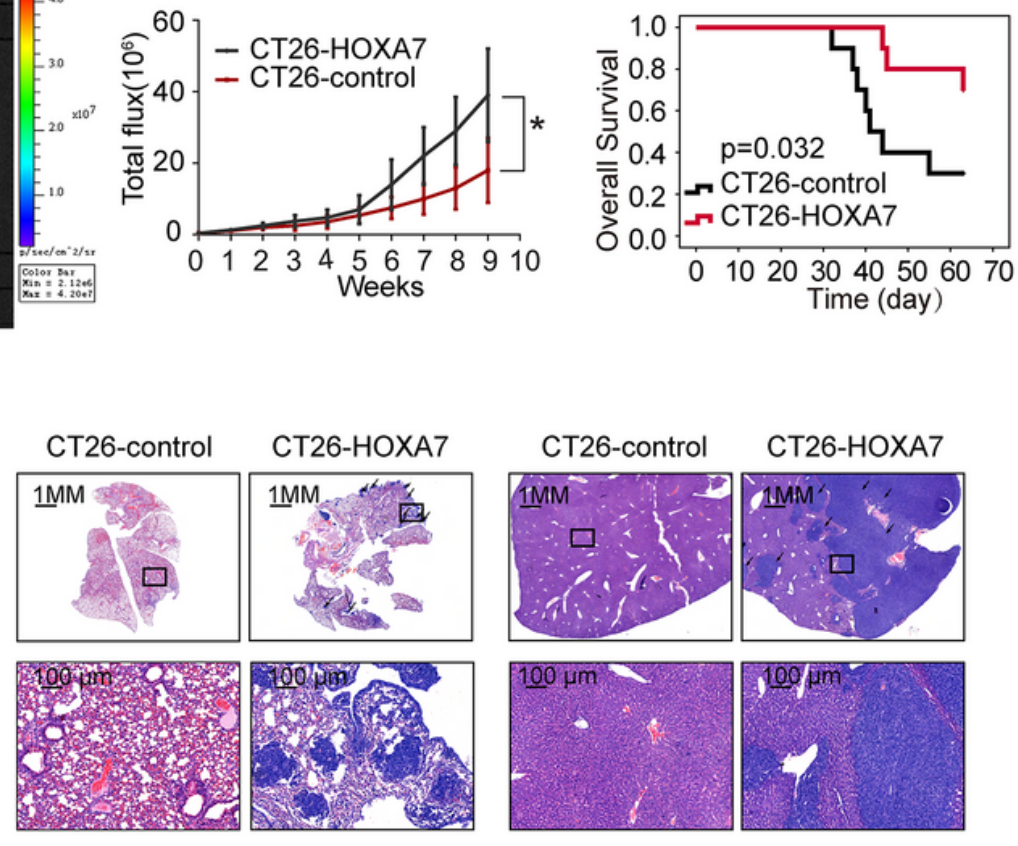

\section{Figure 2}

HOXA7 upregulation promotes KRAS mutant CRC metastasis in immunocompetent mice. (A) . Western blot analyzes HOXA7 expression. (B) . In vivo assays showed that HOXA7 overexpression can promote KRAS mutant CRC metastasis. Bioluminescent images (B). Bioluminescent signals (C). Overall survival (D). The incidence of lung and liver colonization (E). The number of lung and liver colonization (F). HE staining was applied to exhibit metastatic lung and liver nodules(G). ${ }^{\star} \mathrm{P}<0.05$. 

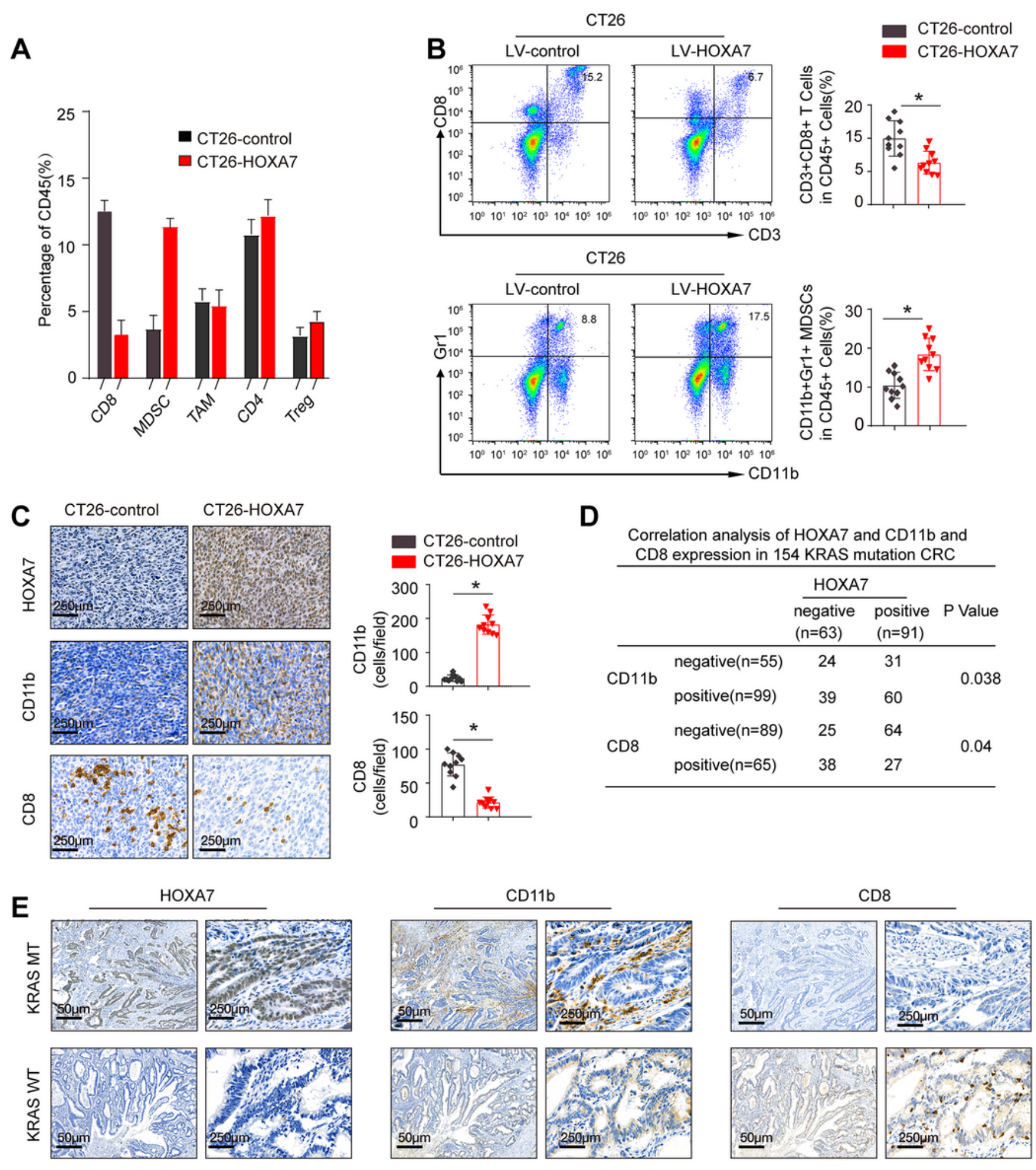

\section{Figure 3}

HOXA7 promotes KRAS-mutant CRC metastasis by recruitment of MDSCs. (A-B). The infiltration of immune cells in the two groups was analyzed by flow cytometry. (C). The infiltration of MDSCs and CD8 in two group was analyzed by IHC. (D). The correlation between HOXA7 expression and the expression of CD11b or CD8 in KRAS mutant CRC tissues. $\neg(E)$. IHC staining showed HOXA7, CD11b and CD8 expression in KRAS mutant CRC samples. 
A

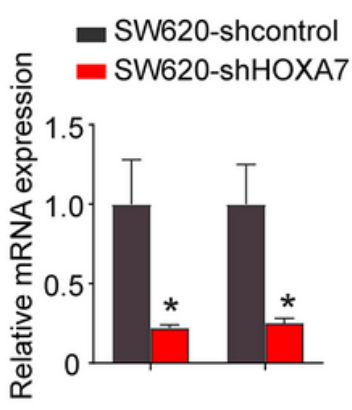

C

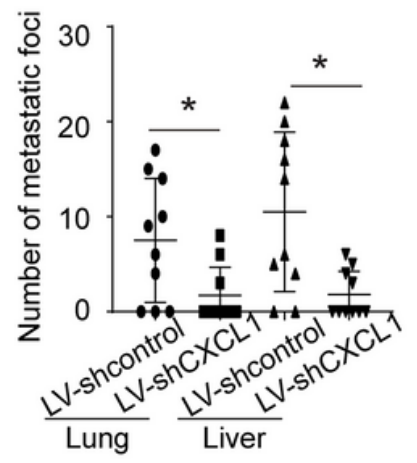

$\mathbf{F}$
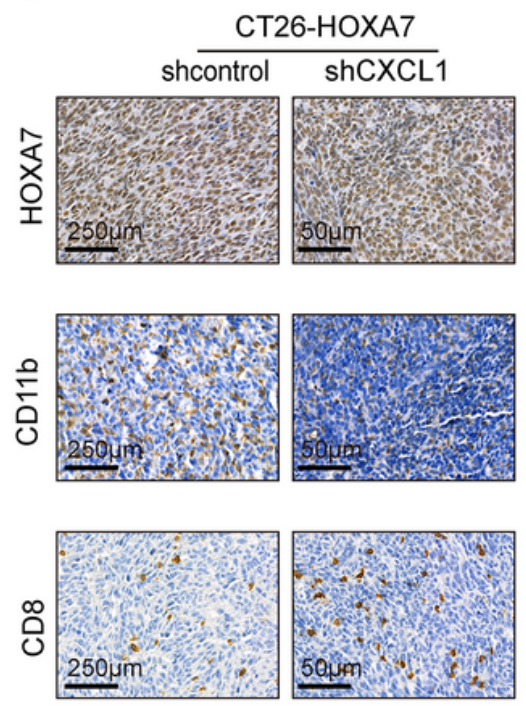

HCT116-shcontrol

- HCT116-shHOXA7

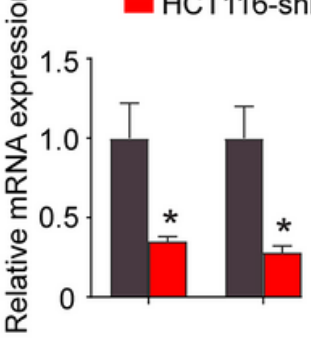

D
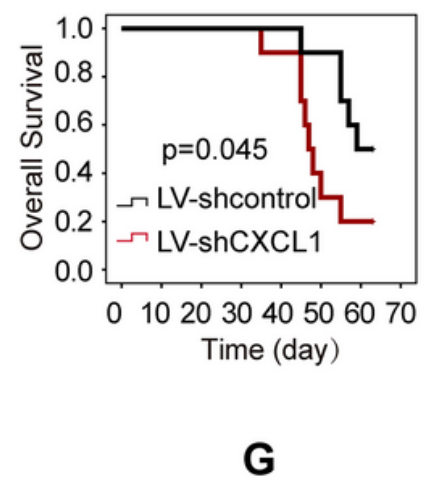

LV-shcontrol LV-shCXCL1
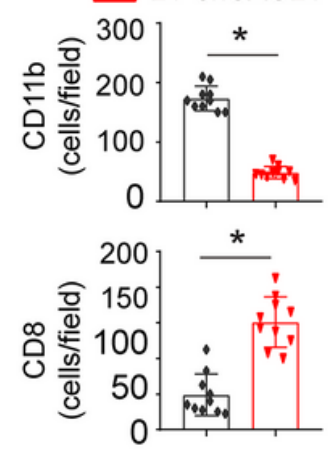

E
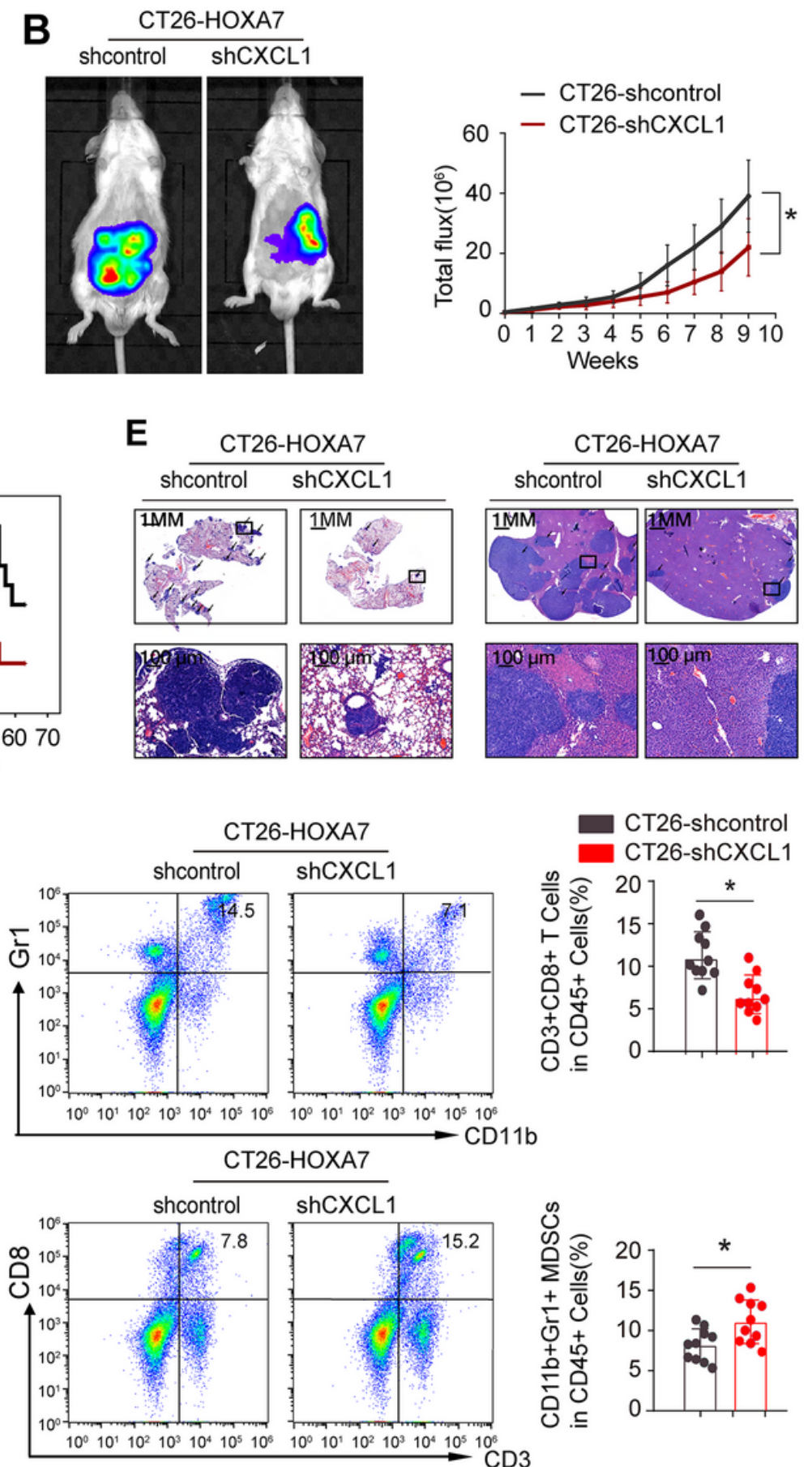

\section{Figure 4}

Downregulation of CXCL1 can inhibit HOXA7-mediated KRAS mutant CRC metastasis. (A). HOXA7 and CXCL1 expression in the indicated cell by Real-time PCR analysis. (B-D). CXCL1 knockdown can reduce HOXA7-mediated KRAS mutant CRC metastasis. Bioluminescence images and Bioluminescence signals (B). The number of lung and liver colonization (C). Overall survival (D). HE staining (E). (F). The infiltration of MDSCs and CD8 in two group was analyzed by IHC. (G). The infiltration of MDSCs and CD8 in two group was analyzed by flow cytometry. All the data are shown as the mean \pm s.d. ${ }^{*} \mathrm{P} \otimes 0.05$. 


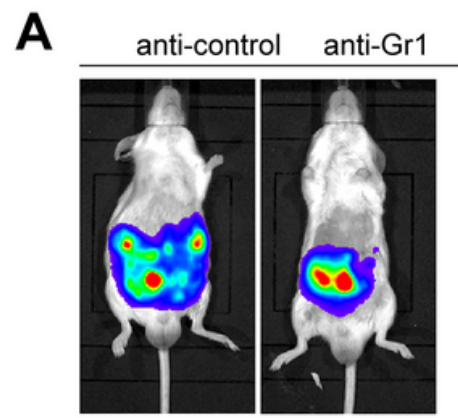

D

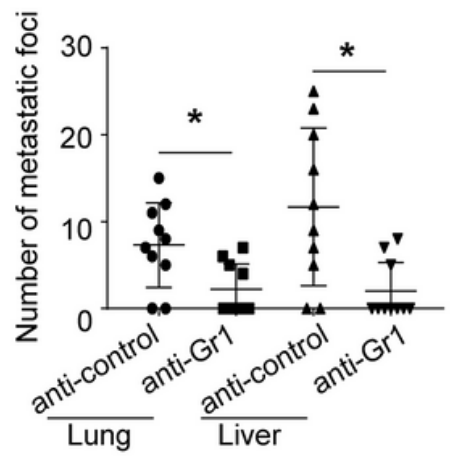

B

Incidence of Lung and Liver Metastasis

\begin{tabular}{lrr}
\hline & Lung & Liver \\
\hline anti-control & $7 / 10$ & $8 / 10$ \\
anti-Gr1 & $3 / 10$ & $4 / 10$
\end{tabular}

$\mathbf{E}$

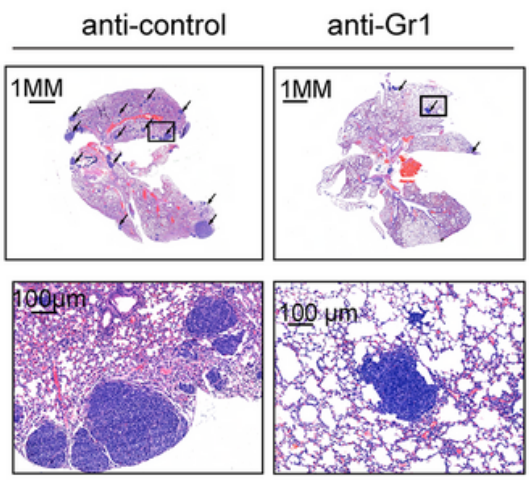

C

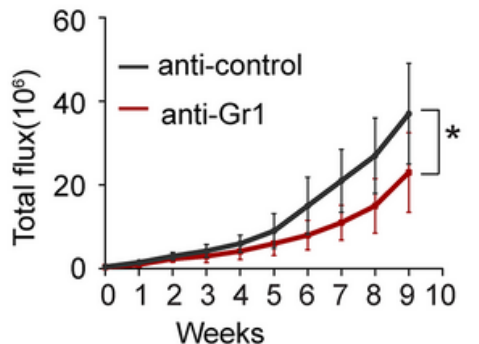

\section{Figure 5}

Depletion of MDSCs by anti-Gr1 can decrease HOXA7-indcued KRAS mutant CRC metastasis. (A-E). In vivo administration of anti-Gr1 neutralizing monoclonal antibody (clone RB6-8C5, 200 $\mu \mathrm{g} / \mathrm{mouse}$, i.p, every 3 day) or isotype control until treatment endpoint. Anti-Gr1 can significantly inhibited HOXA7 mediated KRAS mutant CRC metastasis. Bioluminescence images and the incidence of lung and liver metastasis (A). Bioluminescence signals $(B)$. Overall survival $(C)$. The numbers of lung and liver nodules (D). Representative HE staining (E). The scale bars represent $1 \mathrm{~mm}$ (low magnification) and $100 \mu \mathrm{m}$ (high

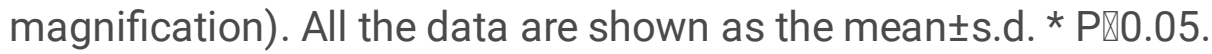


A

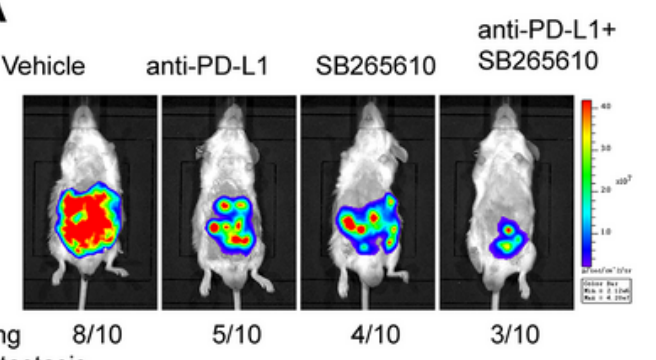

Lung $\quad 8 / 10$

metastasis

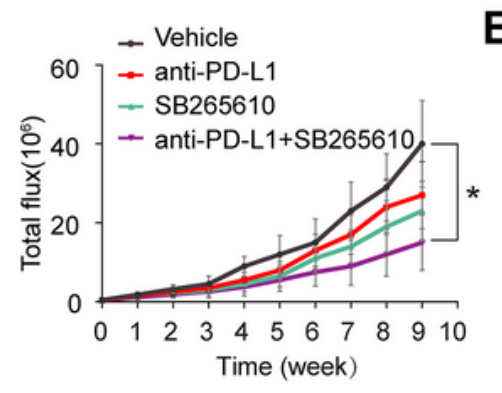

B

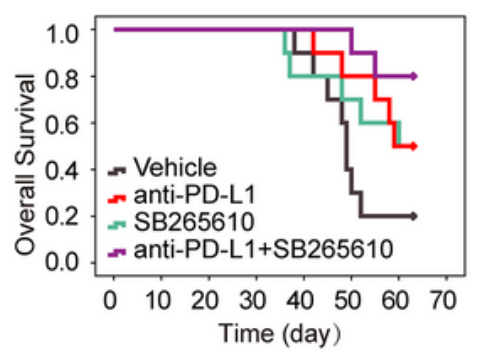

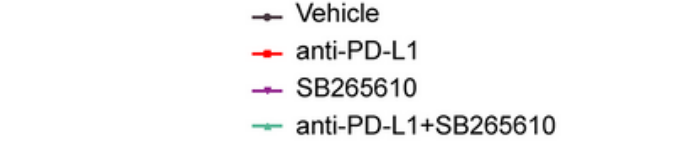

C

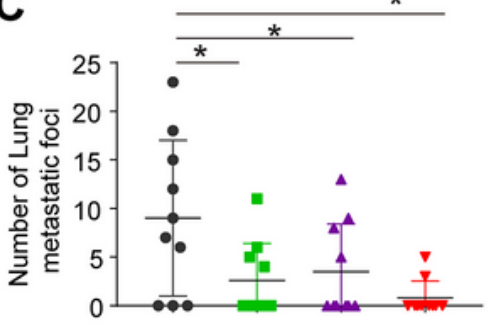

E

Eehicle

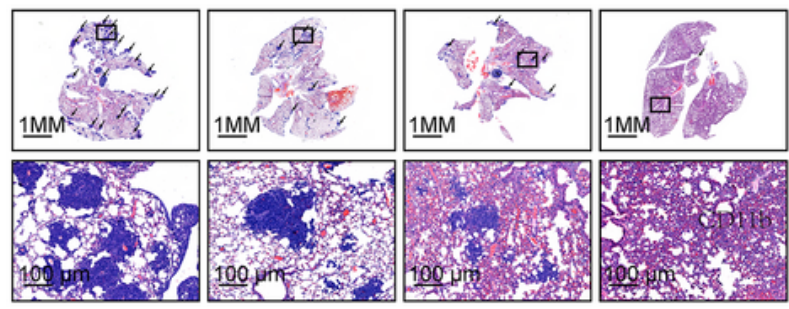

$\mathbf{F}$

anti-PD-L1
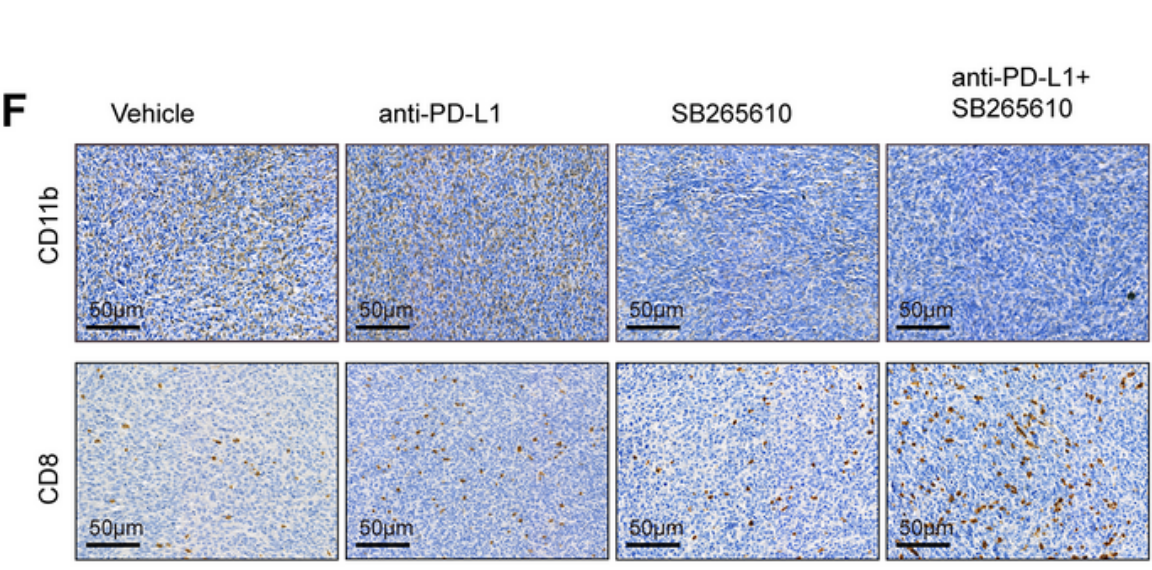

$\rightarrow$ Vehicle

- anti-PD-L1

- SB265610

- anti-PD-L1+SB265610

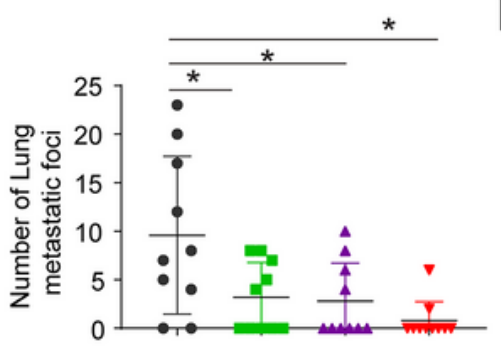

D

\begin{tabular}{ccc}
\multicolumn{3}{c}{ Incidence of Lung Metastasis in } \\
Transplanted Nude mice \\
\hline & Lung & Liver \\
\hline Vhehicle & $8 / 10$ & $7 / 10$ \\
anti-PD-L1 & $5 / 10$ & $4 / 10$ \\
SB265610 & $4 / 10$ & $4 / 10$ \\
anti-PD-L1+SB265610 & $2 / 10$ & $2 / 10$ \\
\hline
\end{tabular}

anti-PD-L1+SB265610 2/10
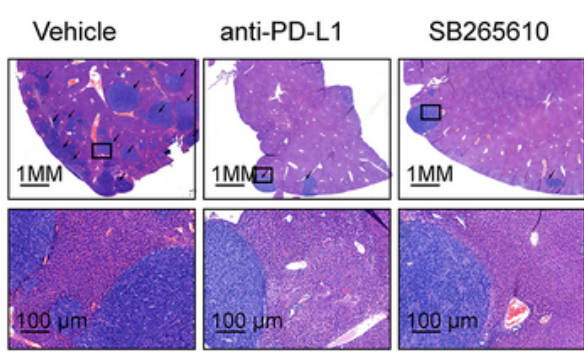

anti-PD-L1+ SB265610
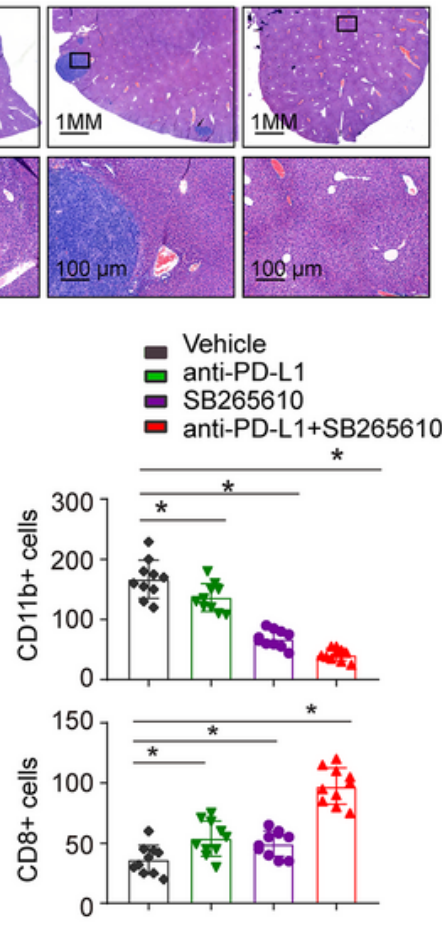

Figure 6

Combined application of CXCR2 inhibitor SB265610 and anti-PD-L1 dramatically blocks HOXA7promoted KRAS-mutant CRC metastasis. (A-E) One week after injection of CT26-HOXA7 cells, mice in four group were treated with vehicle, PD-L1 antibody or/and SB265610 ( $\mathrm{n}=10$ mice/group) until treatment endpoint. In vivo assays showed that combined treatment of PD-L1 antibody and CXCR2 inhibitors can almost block KRAS mutant CRC metastasis totally. Representative bioluminescent images and 
bioluminescent signals in indicated group (A). Overall survival in indicated group (B). The incidence of lung and liver nodules in indicated group $(\mathrm{C})$. The incidence of lung and liver nodules in indicated group(D). Representative HE staining of lung and liver tissues (E). IHC staining detected the infiltration of MDSCs or CD8+ cells in indicated group (F). All the data are shown as the mean \pm s.d. * P®0.05. 